\title{
Health Care Resource Utilization and Cost Before Initial Schizophrenia Diagnosis
}

\author{
Anna Wallace, PhD, MPH; John Barron, PharmD; Whitney York, MS; Keith Isenberg, MD; \\ Jessica Franchino-Elder, MPH, PhD; Matthew Sidovar, MSc, MA; and Michael Sand, PhD, MPH
}

\begin{abstract}
BACKGROUND: The management of schizophrenia, a chronic, multifaceted mental health condition, is associated with considerable health care resource utilization (HCRU) and costs. Current evidence indicates that a high-risk and costly prodromal period, during which patients are likely symptomatic, precedes diagnosis. Better characterization and disease management during this stage could help to improve patient outcomes.
\end{abstract}

OBJECTIVE: To describe and compare HCRU and costs for up to 5 years before diagnosis in a cohort with schizophrenia versus a demographically matched cohort without schizophrenia in a commercially insured U.S. population.

METHODS: This retrospective study identified newly diagnosed schizophrenia patients using enrollee claims in the HealthCore Integrated Research Database between January 1, 2007, and April 30, 2016. The index date was defined as the date of the first medical claim with a schizophrenia diagnosis code. Schizophrenia patients were directly matched (1:4) by age, sex, and region to comparators without schizophrenia who were assigned the same index dates as their matched schizophrenia counterparts. Observation periods were $0-12,13-24,25-36,37-48$, and 49-60 months before the index date. Outcomes included HCRU and costs for inpatient admissions, emergency room visits, outpatient care (office visits and other outpatient services), and medications. Means, standard deviations, medians, and $95 \%$ confidence intervals were calculated for continuous variables; relative frequencies and percentages were calculated for categorical variables. Cohorts were compared with t-tests for continuous variables and chi-square tests for categorical variables. Differences across cohorts were estimated with individual generalized linear models for each observation period, controlling for gender, age, geographic region of residence, health plan type and subscriber status, behavioral pre-index comorbidities and chronic comorbidities during the period before diagnosis.

RESULTS: 6,732 schizophrenia patients were matched to 26,928 patients without schizophrenia. All-cause inpatient admissions were more prevalent among schizophrenia patients than their comparators for all time periods (49-60 months prediagnosis: $9 \%$ vs. $4 \%$; 0-12 months prediagnosis: $33 \%$ vs. $4 \%$ ). The schizophrenia cohort had higher adjusted all-cause per-patient per-month health care costs relative to comparators from the earliest period of 49-60 months prediagnosis ( $\$ 557$ [95\% $\mathrm{Cl}=474-639]$ vs. $\$ 321[95 \% \mathrm{Cl}=288-355])$ through 0-12 months prediagnosis $(\$ 1,058[95 \%$ $\mathrm{Cl}=998-1,115]$ vs. $\$ 338$ [95\% Cl=320-355]). Behavioral health-related costs were different in each time period as were cost ratios (schizophrenia costs: comparator costs), which increased from 5.4 in the earliest period to 14.8 in the year before diagnosis.

CONCLUSIONS: Schizophrenia patients had higher all-cause and behavioral health-related HCRU and costs before diagnosis than matched controls. Costs increased from 5 years to 1 year prediagnosis for schizophrenia patients driven primarily by inpatient hospital stays and prescription drug costs, but remained stable for comparators. Additional research is needed for the development of predictive models to aid in the identification of highrisk patients.

J Manag Care Spec Pharm. 2019;25(10):1102-10

Copyright $\odot 2019$, Academy of Managed Care Pharmacy. All rights reserved.

\section{What is already known about this subject}

While schizophrenia is less common than other mental health conditions such as depression and anxiety, it has a debilitating effect on $0.6 \%-1.9 \%$ of the U.S. population; the annual diagnosed prevalence of the disease is estimated at 5.1 per 1,000 lives.

Schizophrenia exacts a large clinical burden on patients, dramatically reduces their ability to function in society, diminishes the productivity of patients and family memberswho are often their primary caregivers - and is associated with a range of physical and mental disabilities.

The economic burden attributable to this disease is large and increasing rapidly-growing from an estimated $\$ 62.7$ billion in 2002 to $\$ 155.7$ billion in 2013 in the United States because of myriad interrelated contributory factors.

\section{What this study adds}

From as early as 5 years before a schizophrenia diagnosis, health care resource utilization-including behavioral health-related inpatient admissions-emergency room visits, and outpatient visits were higher among the schizophrenia cohort than individuals without schizophrenia.

During all of the prediagnostic observation periods, patients subsequently diagnosed with schizophrenia had higher health care costs compared with matched individuals without schizophrenia; for schizophrenia patients, costs peaked in the year before diagnosis while they remained unchanged for individuals without schizophrenia.

In this high-cost phase of a costly disease, knowledge that costs were mainly driven by greater proportions of emergency room visits, inpatient hospitalizations, and higher behavioral healthrelated pharmacy costs could optimize the financial management of patient care.

S chizophrenia is a mental health disease distinguishable by chronic psychotic symptoms that are uncommon in the general population. ${ }^{1,2}$ Prevalence in the United States ranges from $0.6 \%$ to $1.9 \% .^{3}$ A 2002 study estimated the annual prevalence of diagnosed schizophrenia at 5.1 per 1,000 lives. ${ }^{4}$ The American Psychiatric Association reports no gender difference in prevalence, however, males experience the onset of schizophrenia symptoms at an earlier age, generally in their 
20 s versus late 20 s or early 30 s for females. ${ }^{2,5}$ Cloutier et al. (2016) estimated the costs of schizophrenia at $\$ 155.7$ billion in 2013. ${ }^{6}$ Unemployment (38\%), productivity loss associated with caregiving (34\%), and direct health care costs (24\%) were the largest contributors. ${ }^{6,7}$

In the period before a schizophrenia diagnosis, patients are in a clinically high-risk state for conversion to psychosis. Patients are believed to experience attenuated psychosis syndrome, a recent inclusion in the Diagnostic and Statistical Manual of Mental Disorders, Fifth Edition (DSM-5). ${ }^{5}$ Patients exhibit subclinical symptoms of psychosis and have a reduced quality of life compared with healthy individuals during this time. ${ }^{8,9}$ The absence of data on health care resource utilization (HCRU) and costs before diagnosis limits the understanding of this period and the ability to identify patients who may subsequently be diagnosed with schizophrenia.

It has been hypothesized that early treatment before the first episode of psychosis (FEP) may prevent or delay disease onset, improve quality of life, and reduce the economic burden of the illness. ${ }^{9,10}$ In 1 small study and an associated longer term follow-up in the Netherlands, clinically high-risk patients for FEP who received cognitive behavioral therapy in addition to routine therapy had significantly lower incidence of FEP compared with patients receiving routine therapy alone, at 18-month, and 4-year follow-up periods. ${ }^{11-13}$

Most available studies have focused primarily on the HCRU and costs for incident and prevalent schizophrenia patients. ${ }^{7,14,15}$ This points to a need for new insights on resource use and costs during the period preceding schizophrenia diagnosis.

The objectives of this study were to describe the HCRU and costs for schizophrenia patients for up to 5 years before diagnosis compared with a demographically matched comparator cohort, selected from the general population of patients without schizophrenia, and to estimate adjusted costs during the same time periods.

\section{Methods}

\section{Study Design}

This U.S.-based, retrospective cohort study used medical and pharmacy claims to identify newly diagnosed schizophrenia patients in the HealthCore Integrated Research Database between January 1, 2007, and April 30, 2016. The index date was defined as the earliest inpatient admission date or emergency room (ER)/office visit service date with a schizophrenia diagnosis code. The database is a repository of administrative claims from 14 geographically dispersed health plans in the United States. This observational study was exempt from institutional review board informed consent requirements. Researchers accessed a limited dataset, without patient identifiers, and reported summary statistics. The study complied with the Health Insurance Portability and Accountability Act (HIPAA).

\section{Inclusion Criteria}

Patients with $\geq 1$ inpatient/ER claim with primary diagnosis of schizophrenia (International Classification of Diseases, Ninth/ Tenth Revision, Clinical Modification [ICD-9-CM/ICD-10-CM]; ICD-9-CM: 295.xx, ICD-10-CM: F20\%) or $\geq 2$ office visit claims with diagnosis of schizophrenia in any position or $\geq 2$ claims in any setting with diagnosis of schizophrenia in any position plus Current Procedural Terminology codes for psychiatric services/office visit evaluation and management codes on the same claim were included. For patients identified using 2 qualifying claims, a minimum of 30 days between claims was required. Included patients were aged 18-54 years on the diagnosis date, had $\geq 12$ months of prediagnosis medical and pharmacy eligibility, and $\geq 30$ days postdiagnosis medical and pharmacy eligibility.

\section{Exclusion Criteria}

Patients were excluded if they had $\geq 1$ medical claim with diagnosis codes for schizophrenia during the pre-index period, which varied in duration depending on the length of continuous eligibility for a given patient but required a minimum of 12 months. Also excluded were patients with $\geq 1$ medical claim with diagnosis codes for neurocognitive disorders including Alzheimer disease, age-related cognitive decline, Pick disease, senile dementia, vascular dementia, diffuse Lewy body disease, and Parkinson disease. The ICD9-CM and ICD-10-CM diagnosis codes used for neurocognitive disorders are ICD-9-CM: 294.1x, 294.9x, 331.83, 290.40, and 799.59; ICD-10-CM: F02.8\%, G31.84\%, F01.5\%, and R41.9\%.

\section{Matching}

Schizophrenia patients were exactly matched using a 1:4 ratio to a comparator cohort based on age, sex, and region of residence. Comparators were assigned the index date of their matched schizophrenia counterpart. All patients were assessed retrospectively from the index date to start of health plan eligibility or the start date of study period, whichever occurred nearest to the index date.

Observation periods were 0-1 year, $>1-2$ years, $>2-3$ years, $>3-4$ years, and >4-5 years pre-index. Only patients with continuous medical and pharmacy eligibility during the time period of interest were included.

\section{HCRU and Costs}

All-cause and behavioral health-related HCRU and costs were described for each of the patient observation periods, and included inpatient admissions, ER visits, office visits, other outpatient services, and medications. Costs included planpaid and patient-paid amounts and were adjusted to 2016 U.S. dollars per the medical care price index information from the Bureau of Labor Statistics. ${ }^{16}$ Medical claims were considered behavioral health-related if behavioral health ICD-9-CM or ICD-10-CM diagnosis codes were present in any position 
(ICD-9-CM: 290-319.xx, 780.0-780.5, and 781.1; ICD-10-CM: F01-F99 and R40-R46). Pharmacy claims for medications with behavioral health indications were considered behavioral health-related and included mood stabilizers, antidepressants, anxiolytics, stimulants, alpha adrenergic agonists, hypnotic/ sedatives, sleep disorder agents, and antipsychotics.

\section{Statistical Methods}

All variables were described using means, standard deviations (SDs), medians, and 95\% confidence intervals (CIs) for continuous variables, and relative frequencies and percentages for categorical variables. Demographics were compared across cohorts using t-tests for continuous variables and chi-square tests for categorical variables. Generalized linear models (gamma distribution, log link) were used to estimate differences across cohorts in annual all-cause and behavioral health-related costs controlling for gender, age, geographic region of residence, health plan type, health plan subscriber status, behavioral related pre-index comorbidities (bipolar disorder, depression, anxiety disorder, attentiondeficit/hyperactivity disorder, substance use disorder, alcohol use disorder, emotional disturbance, suicide/self-inflicted injury, learning disability, and intellectual disability) and chronic comorbidities (dyslipidemia, hypertension, diabetes, and obesity) during the 1-year time period before the period of interest. A unique model was created for each of the patient observation periods ( $0-1$ year, $>1-2$ years, $>2-3$ years, $>3-4$ years, and $>$ 4-5 years pre-index).

\section{Results}

\section{Demographics}

A total of 6,732 newly diagnosed schizophrenia patients were matched to 26,928 patients without schizophrenia, as shown in Figure 1. The number of patients with longer durations of prediagnosis eligibility is indicated in Table 1.

\section{Schizophrenia Cohort}

A greater proportion (57.4\%) of schizophrenia patients were male. The mean age (SD) was 32.8 (12.1) years. A majority of women $(54.9 \%)$ were aged $>35$ years at diagnosis, while a majority of men $(67.9 \%)$ were aged $<35$ years. Proportions by age groups were $15-17$ years (7.3\%), 18-25 years (39.8\%), and 26-35 years (20.8\%). Schizoaffective disorder was the most common type (49.0\%) followed by paranoid (24.4\%) and unspecified (18.7\%) schizophrenia. Disease types were similar between the sexes. The mean (SD) Elixhauser Comorbidity Index (ECI) score was 2.1 (1.9), 2.4 (2.0) for females, and 1.9 (1.8) for males, as shown in Table 1.

\section{Schizophrenia Versus Comparator Cohort}

Health plan type, patient's relationship to subscriber, and median household income were different between the cohorts
$(P<0.001)$. The schizophrenia cohort had a larger proportion of dependent subscribers than the comparator cohort (33.2\% vs. 29.2\%). The mean (SD) ECI was greater for the schizophrenia cohort compared with the comparator cohort, 2.1 (1.9) vs. 0.47 (1.0); $P<0.001$. A smaller proportion of the schizophrenia cohort had an ECI of zero versus the comparator cohort (19.6\% vs. $71.8 \%$ ), as shown in Table 1 .

\section{All-Cause HCRU}

The schizophrenia cohort had more all-cause HCRU versus the comparator group for all the time periods evaluated. The largest differences between the 2 cohorts were observed in inpatient admissions and ER visits. All-cause patient admissions increased from $8.7 \%$ to $32.7 \%$ while ER visits increased from $19.2 \%$ to $32.5 \%$ in the 5 years before diagnosis. For the matched comparator cohort, inpatient admissions remained unchanged from $3.6 \%$ to $3.6 \%$, while ER visits increased from $10.7 \%$ to $12.1 \%$. Patients in the schizophrenia cohort had a higher rate of inpatient hospitalizations (32.7\%) than the comparators (3.9\%). In the 0-1 year prediagnosis period, patients in the schizophrenia cohort had approximately 3-fold more all-cause ER visits than comparators ( $32.5 \%$ vs. $11.9 \%$ ).

\section{Behavioral Health-Related HCRU}

For all periods before diagnosis, behavioral health-related utilization was greater in the schizophrenia cohort. In the schizophrenia cohort, behavioral health-related inpatient admissions increased more than 4 -fold from $6.8 \%$ to $31.1 \%$, ER visits more than tripled, from $7.0 \%$ to $21.9 \%$, and officebased outpatient visits increased substantially from $24.3 \%$ to 45.5\%; rates in the comparator cohort remained constant: $0.9 \%$ to $1.2 \%$ for inpatient admissions, $1.7 \%$ to $2.8 \%$ for ER visits, and $10.3 \%$ to $12.8 \%$ for office-based outpatient visits. The proportion of schizophrenia patients seeing a psychiatrist or psychologist increased from 26.3\% (89\% of whom were seen by a psychiatrist) in the $>4-5$ year period to $45.2 \%$ in the year before diagnosis. The largest increases in HCRU were seen during the year before diagnosis, when nearly three quarters of the schizophrenia patients had behavioral health-related outpatient visits compared with only $18.2 \%$ of the comparator cohort. Inpatient admissions in the year before diagnosis in the schizophrenia cohort were substantially greater than the comparator cohort ( $31.1 \%$ vs. $1.2 \%$ ). ER admissions in the schizophrenia cohort were nearly 10 times that in the comparator group, $21.9 \%$ vs. $2.8 \%$, respectively. Nearly half $(42.5 \%, n=3,043)$ of the schizophrenia patients were seen by a psychiatrist or psychologist in the outpatient setting in the year before diagnosis (compared with 3.8\% of comparators); of the schizophrenia patients, $92 \%(n=2,790)$ were seen by psychiatrists. 
Patients with $\geq 1$ claim with ICD-9/10-CM diagnosis code for schizophrenia (ICD-9-CM: 295.xx; ICD-10-CM: F20\%) between January 1, 2007, and April 30, 2016 $\mathrm{N}=56,733$

Patients with $\geq 1$ inpatient/ER claim with primary diagnosis of schizophrenia OR $\geq 2$ office visit claims with diagnosis of schizophrenia in any position OR

$\geq 2$ claims with schizophrenia diagnosis in OUT setting with diagnosis claims in office setting or psychiatric services in OUT setting OR

$\geq 2$ claims with schizophrenia diagnosis and psychiatric services in any setting between January 1,2007 , and April 30, 2016 (earliest claim =index date; newly diagnosed patients) $\mathrm{n}=30,156$

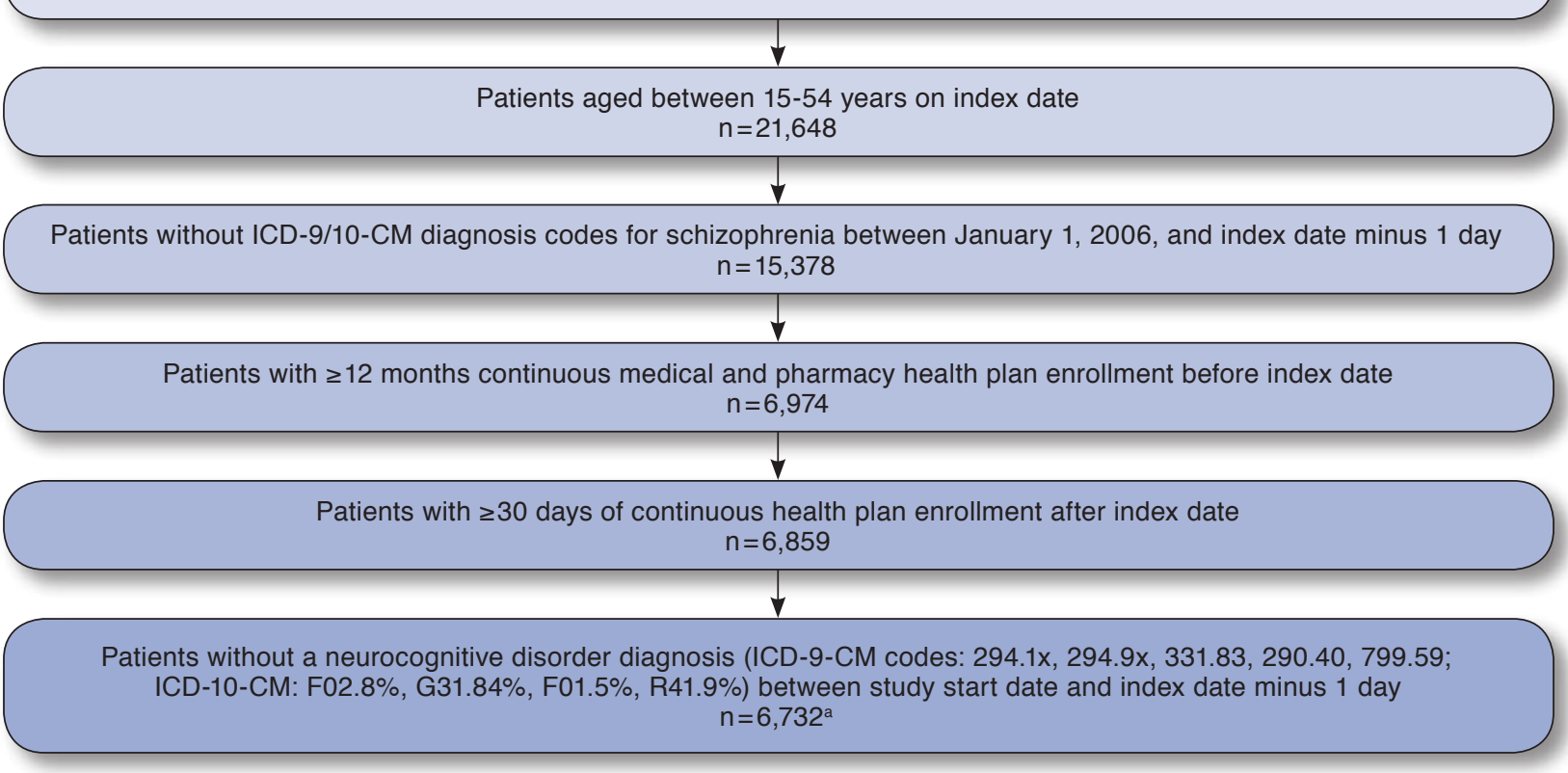

ane patient without continuous eligibility around index date was excluded from the final schizophrenia cohort. $E R=$ emergency room; ICD-9/10-CM = International Classification of Diseases, Ninth/Tenth Revision, Clinical Modification.

\section{All-Cause Costs}

The average allowed unadjusted healthcare costs (plan paid + patient paid per patient per month; PPPM) are shown in the Appendix (available in online article). As early as 5 years before diagnosis, the PPPM all-cause health care costs in the schizophrenia cohort were $\$ 712$, while in the demographically similar cohort without schizophrenia costs were $\$ 278$. For the schizophrenia cohort, the total all-cause costs increased by $\$ 464$ PPPM over the study period (from $\$ 712$ at $>4-5$ years to $\$ 1,176$ PPPM at 1 year before diagnosis); costs in the comparator cohort varied little over time (from $\$ 278$ at $>4-5$ years to $\$ 317$ PPPM at 1 year before diagnosis). During the 1-year period before diagnosis, the schizophrenia costs were composed of 39\% inpatient costs (\$464), $28 \%$ outpatient (office visits + other outpatient) costs (\$327), 26\% pharmacy costs
(\$301), and 7\% ER costs (\$84). The greatest difference in costs between the schizophrenia and comparator cohorts were for pharmacy (\$301 vs. \$65) and inpatient services (\$464 vs. \$73).

For patients with schizophrenia, the adjusted mean all-cause cost PPPM for each observation period was greater than the mean cost PPPM in the comparator cohort (all adjusted mean cost ratios were greater than $1, P$ values $<0.05$ ), as shown in Figure 2 . The adjusted mean all-cause cost ratios for the period from 5 years to $>2$ years before diagnosis were similar, ranging between 1.70 and 1.76. The adjusted cost ratio, however, increased for the 1- to 2-year period before diagnosis and further increased in the year before diagnosis (cost ratio $[95 \%$ $\mathrm{CI}]=2.10[1.95-2.26]$ vs. $3.13[2.88-3.40])$. The schizophrenia cohort had higher adjusted all-cause PPPM health care costs relative to comparators from the earliest period of $>4$ to 
TABLE 1 Baseline Demographic and Clinical Characteristics

\begin{tabular}{|c|c|c|c|c|c|c|c|}
\hline Characteristic & $\begin{array}{l}\text { Schizophrenia } \\
\text { Cohort }\end{array}$ & $\begin{array}{c}\text { Comparator } \\
\text { Cohort }^{\mathrm{a}}\end{array}$ & $\begin{array}{c}P \\
\text { Value }\end{array}$ & Characteristic & $\begin{array}{c}\text { Schizophrenia } \\
\text { Cohort }\end{array}$ & $\begin{array}{c}\text { Comparator } \\
\text { Cohort }^{\mathrm{a}}\end{array}$ & $\begin{array}{c}P \\
\text { Value } \\
\end{array}$ \\
\hline Number of patients & 6,732 & 26,928 & & \multicolumn{4}{|c|}{ Type of schizophrenia on index date, ${ }^{3} \mathrm{n}(\%)$} \\
\hline Age, mean (SD) & $32.82(12.1)$ & $32.82(12.1)$ & $N A^{b}$ & Schizoaffective disorder & $3,300(49.0)$ & & \\
\hline \multicolumn{3}{|l|}{ Age group on index date, $\mathrm{n}(\%)$} & $\mathrm{NA}^{\mathrm{b}}$ & Other specified types & $159 \quad(2.4)$ & & \\
\hline $15-17$ & $454 \quad(6.7)$ & $1,828 \quad(6.8)$ & & Unspecified & $1,256(18.7)$ & & \\
\hline $18-25$ & $2,137 \quad(31.7)$ & $8,534(31.7)$ & & \multicolumn{3}{|c|}{ Median household incomec for ZIP code by quartile, $\mathrm{n}(\%)$} & $<0.001$ \\
\hline $26-35$ & $1,326(19.7)$ & $5,320(19.8)$ & & lst & $1,687(25.1)$ & $6,478(24.1)$ & \\
\hline $36-45$ & $1,363(20.2)$ & $5,434(20.2)$ & & 2nd & $1,510(22.4)$ & $6,478 \quad(24.1)$ & \\
\hline $46-54$ & $1,452 \quad(21.6)$ & $5,812(21.6)$ & & $3 r d$ & $1,404(20.9)$ & $6,476(24.0)$ & \\
\hline \multicolumn{3}{|l|}{ Sex, n (\%) } & $N A^{b}$ & 4th & $1,608(23.9)$ & $6,479(24.1)$ & \\
\hline $\begin{array}{l}\text { Male } \\
\text { Female }\end{array}$ & $3,866 \quad(57.4)$ & $15,464 \quad(57.4)$ & & \multicolumn{3}{|c|}{ Elixhauser Comorbidity Index, ${ }^{\mathrm{d}} \mathrm{n}(\%)$} & $<0.001$ \\
\hline $\begin{array}{l}\text { Female } \\
\text { Region of residence, } \mathrm{n}(\%)\end{array}$ & \multicolumn{2}{|c|}{ Region of residence, $\mathrm{n}(\%)$} & NAb & 0 & $1,322(19.6)$ & $19,324(71.8)$ & \\
\hline Northeast & $1,175 \quad(17.5)$ & $4,700 \quad(17.5)$ & & $1-2$ & $3,185 \quad(47.3)$ & $6,379(23.7)$ & \\
\hline Midwest & $1,802(26.8)$ & $7,208(26.8)$ & & $3-4$ & $1,532(22.8)$ & $1,007 \quad(3.7)$ & \\
\hline South & $1,966(29.2)$ & $7,864(29.2)$ & & $\geq 5$ & $693(10.3)$ & $(0.8)$ & $<0.001$ \\
\hline West & $1,789(26.6)$ & $7,156(26.6)$ & & \multicolumn{4}{|l|}{ Baseline comorbidities, $\mathrm{n}(\%)$} \\
\hline \multicolumn{3}{|l|}{ Health plan type, n (\%) } & $<0.001$ & Dyslipidemia & $1,138(16.9)$ & $2,947 \quad(10.9)$ & $<0.001$ \\
\hline $\mathrm{HMO}$ & $1,538(22.8)$ & $5,854 \quad(21.7)$ & & Hypertension & $1,156 \quad(17.2)$ & $2,426 \quad(9.0)$ & $<0.001$ \\
\hline $\mathrm{PPO}$ & $4,721 \quad(70.1)$ & $18,105 \quad(67.2)$ & & Ischemic heart disease & $134 \quad(2.0)$ & $(0.8)$ & $<0.001$ \\
\hline Other & $473 \quad(7.0)$ & $2,969 \quad(11.0)$ & & Congestive heart failure & $(0.6)$ & $(0.2)$ & $<0.001$ \\
\hline \multicolumn{3}{|c|}{ Patient's relationship to subscriber, n (\%) } & $<0.001$ & Stroke or transient ischemic & (1.4) & $(0.3)$ & $<0.001$ \\
\hline Self & $3,387(50.3)$ & $14,682(54.5)$ & & & & & \\
\hline Spouse/partner & $1,107(16.4)$ & $4,373(16.2)$ & & $\begin{array}{l}\text { Chronic obstructive } \\
\text { pulmonary disease }\end{array}$ & (2.9) & (0.6) & $<0.001$ \\
\hline Dependent & $2,238 \quad(33.2)$ & $7,873 \quad(29.2)$ & & Asthma & $(8.1)$ & 1,097 & $<0.001$ \\
\hline \multicolumn{4}{|c|}{ Type of schizophrenia on index date, ${ }^{3} \mathrm{n}(\%)$} & Diabetes & $(7.4)$ & $(2.9)$ & $<0.001$ \\
\hline Disorganized & $91 \quad(1.4)$ & & & Overweight/obesity & $474 \quad(7.0)$ & $(3.0)$ & $<0.001$ \\
\hline Catatonic & $69 \quad(1.0)$ & & & \multicolumn{4}{|c|}{ Patient's prediagnosis eligibility in years, n (\%) } \\
\hline Paranoid & $1,643(24.4)$ & & & $\geq 1$ & $6,732(100.0)$ & $26,928(100.0)$ & \\
\hline Schizophreniform disorder & $412 \quad(6.1)$ & & & $\geq 2$ & $4,059(60.3)$ & $16,925(62.9)$ & \\
\hline Latent & $62 \quad(0.9)$ & & & $\geq 3$ & $2,646 \quad(39.3)$ & $11,155 \quad(41.4)$ & \\
\hline \multirow{2}{*}{$\begin{array}{l}\text { Schizophrenic disorder } \\
\text { residual type }\end{array}$} & $110 \quad(1.6)$ & & & $\geq 4$ & $1,826 \quad(27.1)$ & $7,539(28.0)$ & \\
\hline & & & & $\geq 5$ & $1,227(18.2)$ & $5,120(19.0)$ & \\
\hline \multicolumn{8}{|c|}{$\begin{array}{l}\text { a Matched based on age, sex, and region of resi } \\
{ }^{2} P \text { value not obtained for matching variables. }\end{array}$} \\
\hline
\end{tabular}

5 years prediagnosis ( $\$ 557[95 \% \mathrm{CI}=474-639]$ vs. $\$ 321[95 \%$ $\mathrm{CI}=288-355])$ through the period nearest to diagnosis, 0 to 1 year prediagnosis ( $\$ 1,058[95 \% \mathrm{CI}=998-1,115]$ vs. $\$ 338[95 \%$ CI $=320-355]$ ), as shown in Table 2.

\section{Behavioral Health-Related Health Care Costs}

During each of the observation periods before diagnosis, the schizophrenia cohort had higher unadjusted behavioral healthrelated health care costs compared with the comparator cohort (Appendix). The trends were similar to the all-cause costs. For the schizophrenia cohort, the total costs were \$391 for the 4- to 5-year observation period and $\$ 364$ for the 3- to 4-year period versus $\$ 60$ and $\$ 53$ over the same periods in the comparator group. Average total costs started increasing at the 2-3 year period before diagnosis (\$451) and continued to $\$ 833$ in the year before diagnosis. As with all-cause costs, behavioral health-related costs in the comparator cohort varied little over time $(\$ 46-\$ 60)$. As with all-cause costs, the greatest difference between the cohorts in behavioral health-related costs was in the year before diagnosis (schizophrenia $\$ 833$ vs. comparator $\$ 56)$. The greatest differences in costs were observed for inpatient services (schizophrenia \$422 vs. comparator \$24) and pharmacy (schizophrenia \$207 vs. comparator \$12). In the year before diagnosis, the majority of all-cause costs in the 


\section{FIGURE 2 Adjusted All-Cause and Behavioral Health-Related Adjusted Cost Ratios: Schizophrenia Versus Comparator Cohorts}

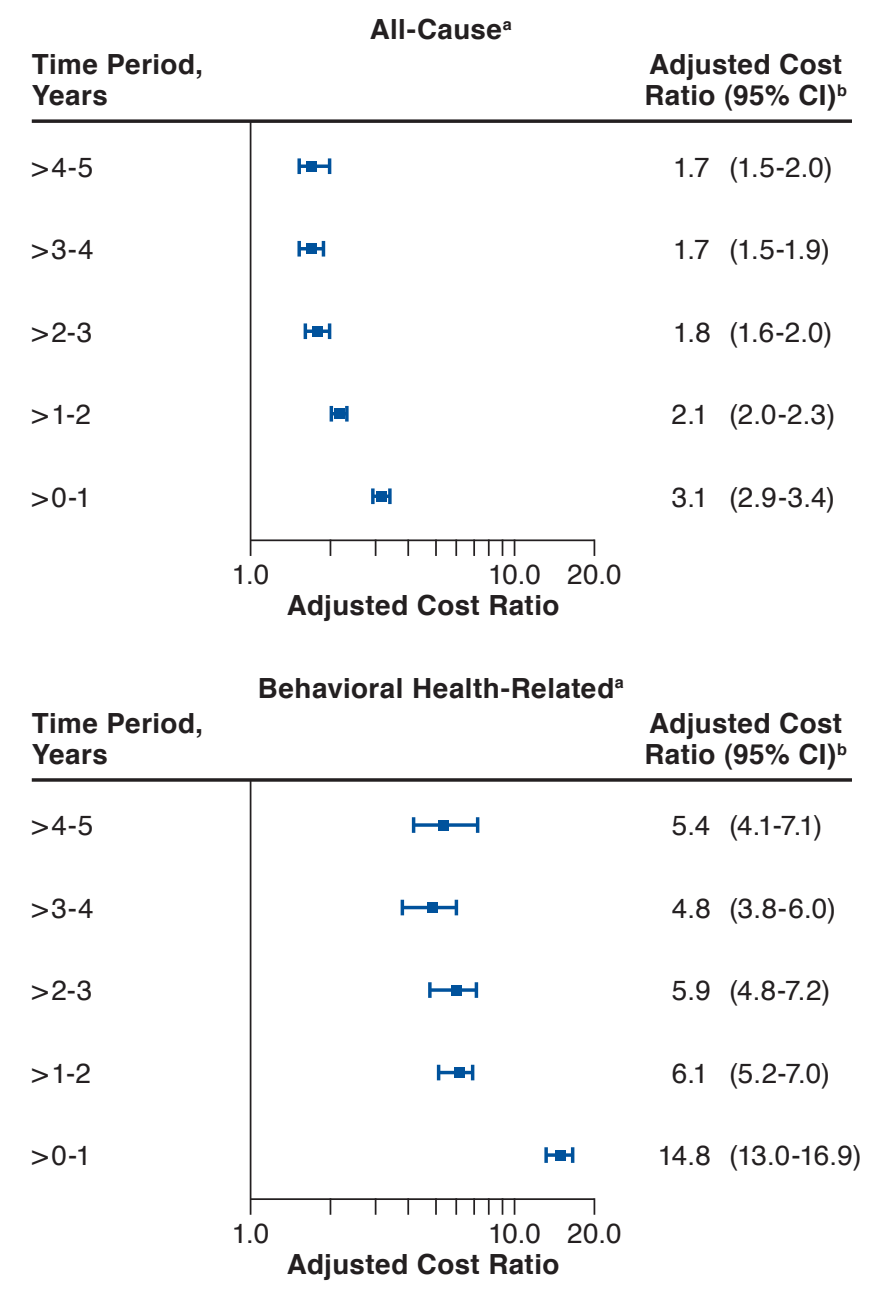

aAll $P$ values $<0.05$; all models adjusted for gender, age, geographic region of patient residence, health plan type, health plan subscriber status, behavioralrelated pre-index comorbidities (bipolar disorder, depression, anxiety disorder, attention deficit hyperactivity, substance abuse disorder, alcohol abuse disorder, emotional disturbance, suicide/self-inflicted injury, learning disability, intellectual disability) and chronic comorbidities (dyslipidemia, hypertension, diabetes, and obesity) during time period before period when costs were estimated.

${ }^{b}$ The cost ratio can be interpreted as follows: over the period of > 4-5 years before diagnosis, adjusted mean all-cause costs among schizophrenia patients were 1.7 times the costs for matched nonschizophrenia patients.

CI= confidence interval.

schizophrenia cohort were attributable to behavioral healthrelated utilization $(70.1 \%$ [\$833/\$1,176]).

For patients with schizophrenia, adjusted mean behavioral health-related cost PPPM for each observation period was greater than the mean cost PPPM in the comparator cohort (all adjusted mean cost ratios were greater than 1; all
$P$ values $<0.01$; Figure 2). The adjusted mean cost ratios from 5 to $>1$ year before diagnosis were similar, ranging between 4.80 and 6.07; the adjusted mean cost ratio increased substantially to 14.82 (95\% CI=13.00-16.89) in the year before diagnosis (Figure 2). The schizophrenia cohort had higher adjusted behavioral health-related PPPM health care costs relative to comparators as early as 5 years before diagnosis ( $>4-5$ years prediagnosis, $\$ 384[95 \% \mathrm{CI}=256-551]$ vs. $\$ 71 \quad[95 \%$ $\mathrm{CI}=51-92])$ through the period most proximal to diagnosis (0-1 year prediagnosis, $\$ 1,189$ [95\% CI=830-1,549] vs. $\$ 80$ [95\% CI $=56-105]$ ), as shown in Table 2 .

\section{Discussion}

This study found that all-cause and behavioral health-related HCRU were greater in the schizophrenia cohort relative to comparators as early as 5 years before diagnosis. While comparator utilization rates remained unchanged over time, in the schizophrenia cohort there were greater levels of all-cause HCRU and costs including increased inpatient admissions and ER visits from the 4-5 year to the 0-1 year observation periods, before initial schizophrenia diagnosis.

The cost patterns persisted following adjustment-mean all-cause and behavioral health-related costs PPPM for each observation period were greater than the mean cost for comparators. The cost ratios were higher from 5 years to $>1$ year before diagnosis and increased in the year before diagnosis. During this time, patients may be in a clinically high-risk state and may experience attenuated psychosis syndrome, ${ }^{5}$ present with subclinical symptoms of psychosis and have quality-of-life deficits relative to counterparts without schizophrenia. ${ }^{8,9}$

The increase in costs during the 5 years before a schizophrenia diagnosis was mainly driven by the increase in pharmacy expenses and inpatient hospitalizations. In the 0-1 years before diagnosis, all-cause inpatient admissions doubled, and behavioral health-related inpatient admissions tripled compared with the 1-2 year period. Increased service utilization, compatible with worsening disease course, weighed on the health care system during the year before diagnosis.

This pattern persisted after variables of interest including age, sex, and prediagnosis comorbidities were adjusted. There was a marked increase in adjusted mean costs for schizophrenia patients from $>1-2$ years before diagnosis to 0-1 year before diagnosis. Major differences in costs between the schizophrenia and comparator cohorts were driven by higher inpatient and pharmacy costs incurred by schizophrenia patients.

To our knowledge, there is no available literature describing the HCRU and costs over this time period for patients before diagnosis of schizophrenia-research has focused primarily on the HCRU and costs for incident and prevalent schizophrenia patients. ${ }^{7,14,15}$ 


\begin{tabular}{|c|c|c|c|}
\hline TABLI & $\begin{array}{l}\text { Adjuste } \\
\text { Health- }\end{array}$ & $\begin{array}{l}\text { All-Cause and } \\
\text { lated Costs }\end{array}$ & Behavioral \\
\hline \multirow[b]{2}{*}{$\begin{array}{l}\text { Observation } \\
\text { Period, Years }\end{array}$} & \multirow[b]{2}{*}{ Cohort } & All-Cause & $\begin{array}{c}\text { Behavioral Health- } \\
\text { Related }\end{array}$ \\
\hline & & $\begin{array}{l}\text { Adjusted Cost, \$ } \\
(95 \% \mathrm{CI})\end{array}$ & $\begin{array}{c}\text { Adjusted Cost, \$ } \\
(95 \% \mathrm{CI})\end{array}$ \\
\hline \multirow[t]{2}{*}{$>4-5$} & Schizophrenia & $557(474-639)$ & $384(256-511)$ \\
\hline & Comparator & $321(288-355)$ & $71 \quad(51-92)$ \\
\hline \multirow[t]{2}{*}{$>3-4$} & Schizophrenia & $544(491-598)$ & $306(251-362)$ \\
\hline & Comparator & $320(297-342)$ & $64 \quad(52-76)$ \\
\hline \multirow[t]{2}{*}{$>2-3$} & Schizophrenia & $605(545-665)$ & $418(339-497)$ \\
\hline & Comparator & $342(316-369)$ & $71 \quad(58-85)$ \\
\hline \multirow[t]{2}{*}{$>1-2$} & Schizophrenia & $690(639-740)$ & $582(420-743)$ \\
\hline & Comparator & $349(325-374)$ & $96 \quad(68-123)$ \\
\hline \multirow[t]{2}{*}{$0-1$} & Schizophrenia & $1,058(998-1,115)$ & $1,189(830-1,549)$ \\
\hline & Comparator & $338(320-355)$ & $80 \quad(56-105)$ \\
\hline
\end{tabular}

Fitch et al. (2014) used medical and pharmacy data between 2007 and 2011 to identify newly diagnosed schizophrenia patients. ${ }^{7}$ The study analyzed annual and longitudinal costs associated with schizophrenia patients in the year following diagnosis. The mean cost (PPPM) for a schizophrenia patient was 4.3 times the mean cost for a demographically adjusted person without schizophrenia ( $\$ 1,806$ vs. \$419). Schizophrenia costs included $42 \%$ inpatient costs, $33 \%$ outpatient costs, and $25 \%$ prescription drug costs. In addition, total patient costs grew from $\$ 800$ per month in the 12 months before the index date (diagnosis) to about $\$ 2,000$ in the month just before diagnosis. These results were similar to our findings; we found the mean cost PPPM for schizophrenia patients during the year before diagnosis to be 3.6 times greater than for patients without schizophrenia (95\% CI=3.3-4.0).

In a study that used administrative medical and pharmacy claims, Nicholl et al. (2010) reported that recently diagnosed schizophrenia patients had hospitalization rates of $22.3 \%$ during the year following diagnosis. ${ }^{15}$ This rate was lower than what we observed in the schizophrenia cohort the year leading up to diagnosis (32.7\%). Nicholl et al. also reported that the mean annual health care costs of recently diagnosed patients were $\$ 20,654$ ( $\sim 1,721$ per month), with inpatient costs making up $62.9 \%$ of total costs. The costs for the newly diagnosed patients were higher than the PPPM estimates in our study for the year leading up to diagnosis $(\$ 1,176)$.

In a large claims-based study in California, Wilson et al. (2011) examined the costs of treating schizophrenia for newly versus previously diagnosed patients. ${ }^{14}$ The study found that the overall direct annual treatment costs and schizophrenia-specific costs were $\$ 12,885$ ( $\sim 1,074$ per month) and $\$ 6,220$ ( $\$ 518$ per month), respectively. The authors also found that inpatient services and medications represented the greatest proportions of schizophrenia costs, as we reported in this study.

Overall, the HCRU patterns in this study could mean that the patients were receiving appropriate care. These patterns, however, could also suggest that patients may not be benefiting from effective coordination of care, evident from their consultations with an array of different providers, and that perhaps better ways to coordinate care and health resource use exist. Irrespective of what the patterns indicate, a clear case could be made for earlier diagnosis and treatment initiation, as prompt identification may lead to timelier care, improved clinical outcomes, and reduced costs.

Research on the management of schizophrenia has focused both on early detection and on delaying the transition to psychosis. Piras et al. (2014) noted that in the course of the past 2 decades, considerable attention was focused on the early detection and prevention of the first psychotic episode in schizophrenia patients. ${ }^{9}$ The ability to detect motor, cognitive, behavioral, and emotional signals has led researchers to hypothesize that earlier intervention in diagnosis and treatment as part of a prevention framework may provide an opening to reduce disease incidence, improve outcomes, and possibly inhibit the emergence of psychotic episodes. The authors noted, however, that while early detection and intervention could prevent or delay disease onset, there is little progress on the emergence of such early treatments currently and further research is required..$^{9}$ Fusar-Poli et al. (2018) concur and note that a major transition in the clinical characterization of psychotic disorders has occurred over the last 2 decades. ${ }^{17}$ The authors point to the emergence of the concept of the clinical high-risk state for psychosis to describe the prepsychotic phase and what could be prodromal symptoms. The authors noted that the high-risk state is gaining in importance and is under consideration as a diagnostic category in the DSM-5. ${ }^{17}$

While the means for early detection and early treatment intervention are still not available, a number of recent studies have focused on how delaying the passage to psychosis has led to a general reduction in the length of untreated psychosis and treatment for mental health services. ${ }^{18-20}$ Among the interventions under consideration are antipsychotic drugs, ${ }^{21}$ cognitive behavioral therapy, ${ }^{20}$ and omega-3 fish oil. ${ }^{18}$ While these interventions appear promising, the answers to a number of operational and outcomes questions remain inconclusive. ${ }^{19}$

The results of this study could be combined with existing data to develop a model to identify high-risk patients. As with currently available data, it is not possible to conclude 
from the results of this study that earlier identification of symptomatic patients could lead to timelier care, improved outcomes, and reduced costs. Our results, however, suggest that earlier initiation of care could be valuable in addressing those objectives.

\section{Limitations}

The results of this study must be viewed alongside some inherent limitations. Information on patient demographic and clinical characteristics was limited to those available in medical claims; unmeasured factors (e.g., body mass index) may be different between the cohorts despite matching. Claims are subject to coding errors; it is possible patients were misclassified into the wrong cohort or inaccurately identified at the time of initial schizophrenia diagnosis. Schizophrenia is a complex and chronic disease; hence, the first recorded claim for schizophrenia could occur after the true onset of the disease. We studied diagnostic claims and utilization over a period of time before diagnosis where patients may exhibit symptoms that are likely to be more general in early stages of disease and become more specific as time to diagnosis gets shorter. Further, the generalizability of these results may be limited to patient populations that have commercial health insurance. Patients evaluated in the observation periods $>1$ year before diagnosis were a subset of the population initially identified. Thus, the results obtained for these observation periods may be subject to selection bias.

\section{Conclusions}

For all of the prediagnosis time periods evaluated in this study, schizophrenia patients had higher all-cause and behavioral health-related HCRU and costs relative to matched comparators without schizophrenia. The increase in costs from 5 years to 1 year prediagnosis for schizophrenia patients was driven primarily by inpatient hospital stays and prescription drug costs, which remained relatively unchanged for comparators without schizophrenia. Our findings also showed that schizophrenia patients had substantial comorbidities preceding the schizophrenia diagnosis. These results may support the hypothesis that early identification could lead to timelier care and may help to improve outcomes and control costs. Additional research could be useful to develop predictive models to aid in the identification of high-risk patients, and should consider including psychiatric comparator groups (bipolar, major depression) to explore whether such a model can discriminate between patients who are subsequently diagnosed with schizophrenia versus individuals who will receive a diagnosis for a different mental health condition.

\section{Authors}

ANNA WALLACE, PhD, MPH; JOHN BARRON, PharmD; and WHITNEY YORK, MS, HealthCore, Wilmington, Delaware. KEITH ISENBERG, MD, Anthem, Indianapolis, Indiana, and Department of Psychiatry, Washington University School of Medicine, St. Louis, Missouri. JESSICA FRANCHINO-ELDER, MPH, PhD; MATTHEW SIDOVAR, MSc, MA; and MICHAEL SAND, PhD, MPH, Boehringer Ingelheim Pharmaceuticals, Ridgefield, Connecticut.

AUTHOR CORRESPONDENCE: John Barron, PharmD, Principal Scientist, HealthCore, 123 Justison St., Ste 200, Wilmington, DE 19801. Tel.: 302.230.2113; E-mail: jbarron@healthcore.com.

\section{DISCLOSURES}

This study was sponsored by Boehringer Ingelheim Pharmaceuticals. Barron is an employee of HealthCore, which received funding from Boehringer Ingelheim to conduct this study. Wallace and York were employed by HealthCore at time of this study. Isenberg is an employee of Anthem. Franchino-Elder, Sidovar, and Sand are employees of Boehringer Ingelheim.

\section{ACKNOWLEDGMENTS}

Bernard B. Tulsi, MSc, Senior Medical Writer at HealthCore, provided writing and other editorial support, and Mayura Shinde, an employee at HealthCore at the time of the study, provided support in project development and study design.

\section{REFERENCES}

1. National Institute of Mental Health. Schizophrenia. Updated May 2018. Available at: https://www.nimh.nih.gov/health/statistics/prevalence/schizophrenia.shtml. Accessed June 10, 2019.

2. Patel KR, Cherian J, Gohil K, Atkinson D. Schizophrenia: overview and treatment options. P T. 2014;39(9):638-45.

3. van Os J, Kapur S. Schizophrenia. Lancet. 2009;374(9690):635-45.

4. Wu EQ, Birnbaum HG, Shi L, et al. The economic burden of schizophrenia in the United States in 2002. J Clin Psychiatry. 2005;66(9):1122-29.

5. American Psychiatric Association. Diagnostic and Statistical Manual of Mental Disorders. 5th ed. Washington, DC: American Psychiatric Association; 2013.

6. Cloutier M, Aigbogun MS, Guerin A, et al. The economic burden of schizophrenia in the United States in 2013. J Clin Psychiatry. 2016;77(6):764-71.

7. Fitch K, Iwasaki K, Villa KF. Resource utilization and cost in a commercially insured population with schizophrenia. Am Health Drug Benefits. 2014;7(1):18-26

8. Fusar-Poli P, Rocchetti M, Sardella A, et al. Disorder, not just state of risk: meta-analysis of functioning and quality of life in people at high risk of psychosis. Br J Psychiatry. 2015;207(3):198-206.

9. Piras S, Casu G, Casu MA, et al. Prediction and prevention of the first psychotic episode: new directions and opportunities. Ther Clin Risk Manag. 2014;10:241-53

10. Fusar-Poli P, Borgwardt S, Bechdolf A, et al. The psychosis high-risk state: a comprehensive state-of-the-art review. JAMA Psychiatry. 2013; 70(1):107-20.

11. Ising HK, Smit F, Veling W, et al. Cost-effectiveness of preventing firstepisode psychosis in ultra-high-risk subjects: multi-centre randomized controlled trial. Psychol Med. 2015;45(7):1435-46. 
12. Ising HK, Kraan TC, Rietdijk J, et al. Four-year follow-up of cognitive behavioral therapy in persons at ultra-high risk for developing psychosis: the Dutch Early Detection Intervention Evaluation (EDIE-NL) trial. Schizophr Bull. 2016;42(5):1243-52.

13. Nieman DH, McGorry PD. Detection and treatment of at-risk mental state for developing a first psychosis: making up the balance. Lancet Psychiatry. 2015;2(9):825-34.

14. Wilson LS, Gitlin M, Lightwood J. Schizophrenia costs for newly diagnosed versus previously diagnosed patients. Am J Pharm Benefits. 2011:3(2):107-15

15. Nicholl D, Akhras KS, Diels J, Schadrack J. Burden of schizophrenia in recently diagnosed patients: healthcare utilisation and cost perspective. Curr Med Res Opin. 2010;26(4):943-55.

16. Bureau of Labor Statistics. CPI-all urban consumers: medical care in United States. Available at: https://data.bls.gov/timeseries/ CUUR0000SAM?output_view=pct_12mths. Accessed June 11, 2019.
17. Fusar-Poli P, De Micheli A, Cappucciati M, et al. Diagnostic and prognostic significance of DSM-5 attenuated psychosis syndrome in services for individuals at ultra high risk for psychosis. Schizophr Bull. 2018;44(2):264-75.

18. Amminger GP, Schäfer MR, Papageorgiou K, et al. Long-chain omega-3 fatty acids for indicated prevention of psychotic disorders: a randomized, placebo-controlled trial. Arch Gen Psychiatry. 2010;67(2):146-54.

19. Cheng SC, Schepp KG. Early intervention in schizophrenia: a literature review. Arch Psychiatr Nurs. 2016;30(6):774-81.

20. van der Gaag M, Nieman DH, Rietdijk J, et al. Cognitive behavioral therapy for subjects at ultrahigh risk for developing psychosis: a randomized controlled clinical trial. Schizophr Bull. 2012;38(6):1180-88.

21. McGorry PD, Yung AR, Phillips LJ, et al. Randomized controlled trial of interventions designed to reduce the risk of progression to first-episode psychosis in a clinical sample with subthreshold symptoms. Arch Gen Psychiatry. 2002;59(10):921-28.

\section{APPENDIX Unadjusted All-Cause and Behavioral Health-Related Health Care Costs (PPPM)}

\section{All-Cause}

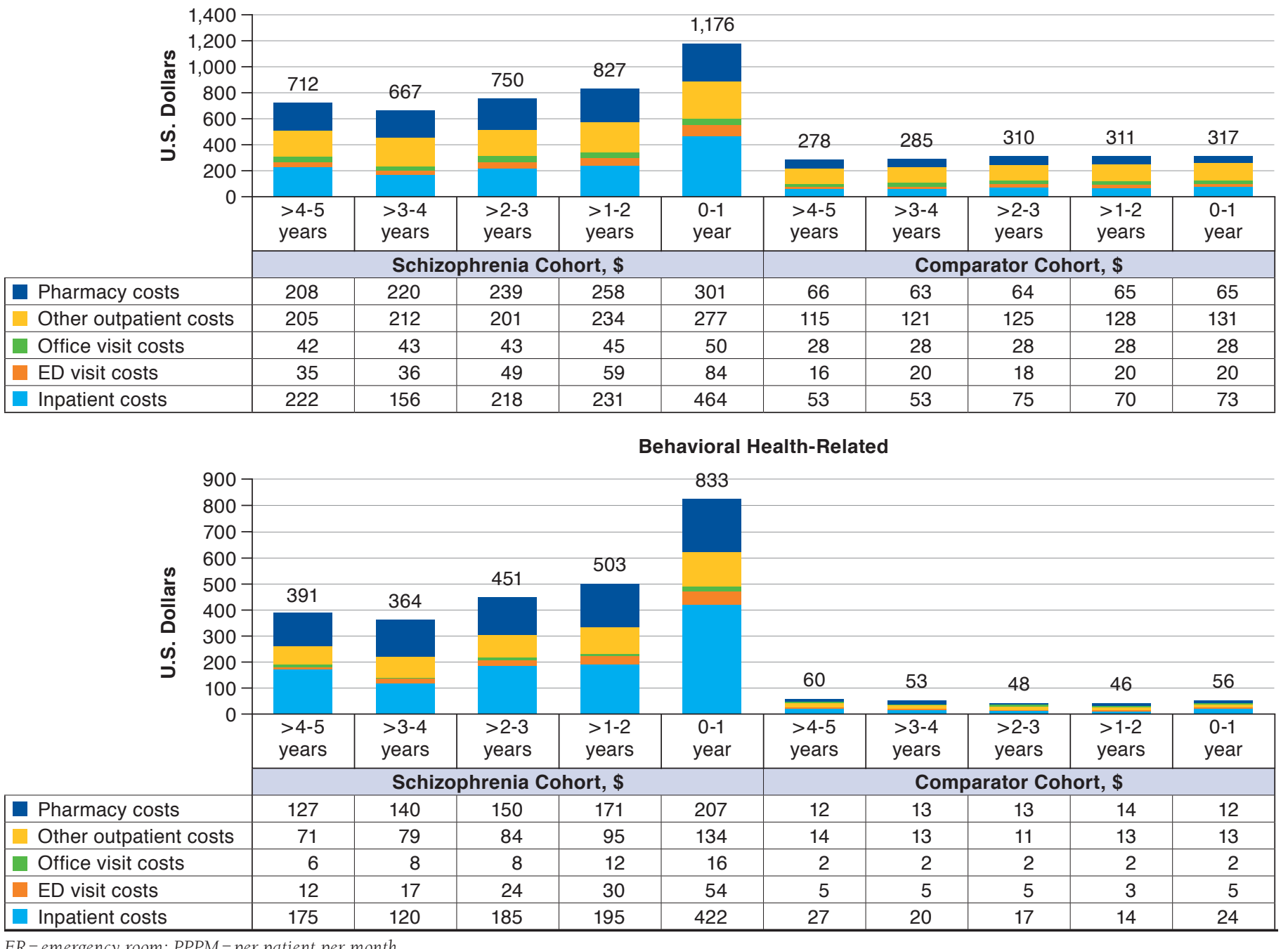

$E R=$ emergency room; $P P P M=$ per patient per month. 